\title{
Tunable Adsorption on Carbon Nanotubes
}

\author{
O. Gülseren, ${ }^{1,2}$ T. Yildirim, ${ }^{1}$ and S. Ciraci ${ }^{3,4}$ \\ ${ }^{1}$ NIST Center for Neutron Research, National Institute of Standards and Technology, Gaithersburg, Maryland 20899-8562 \\ ${ }^{2}$ Department of Materials Science and Engineering, University of Pennsylvania, Philadelphia, Pennsylvania 19104 \\ ${ }^{3}$ Department of Physics, Bilkent University, Ankara 06533, Turkey \\ ${ }^{4}$ Department of Physics, University of Illinois at Chicago, Chicago, Illinois 60607-7059
}

(Received 21 May 2001; published 24 August 2001)

\begin{abstract}
We investigated the adsorption of a single atom, hydrogen and aluminum, on single-wall carbon nanotubes from first principles. The adsorption is exothermic, and the associated binding energy varies inversely as the radius of the zigzag tube. We found that the adsorption of a single atom and related properties can be modified continuously and reversibly by the external radial deformation. The binding energy on the high curvature site of the deformed tube increases with increasing radial deformation. The effects of curvature and radial deformation depend on the chirality of the tube.
\end{abstract}

DOI: 10.1103/PhysRevLett.87.116802

Novel mechanical, electrical, and chemical properties of carbon nanotubes [1-3] have been explored actively with a motivation of finding a new technological application. A single-wall carbon nanotube (SWNT) is usually described by a rolled graphene, where the hexagonal $2 \mathrm{D}$ lattice is mapped on a cylinder of radius $R$ with various helicities characterized by a set of two integers $(n, m)$. A SWNT can display either metallic or insulating electronic structure depending on the helicity and radius [2].

Recent studies [3-7] showed that the electronic properties of SWNTs can be modified by radial deformation. The energy gap of an insulating SWNT can decrease and eventually vanish at an insulator-metal transition with increasing applied radial strain. The density of states at the Fermi energy, $\mathcal{D}\left(E_{F}\right)$, of a metallized SWNT increases with the increasing radial strain. More interestingly, the radial deformation necessary to induce metallicity was found to be in the elastic range. Therefore, all strain induced changes in the electronic and also in mechanical properties are reversible.

Most noticeably, the radial strain disturbs the uniformity of charge distribution. This, in turn, may impose changes in the chemical reactivity and hence in the interaction of tube surface with foreign atoms and molecules. It is therefore anticipated that not only band gap but also chemical reactions taking place on the surface of a SWNT can be engineered through radial deformation. In this paper, we explore this feature by using the predictive power of the density functional theory and demonstrate that indeed adsorption of foreign atoms on carbon nanotubes and associated properties can be modified continuously and reversibly. Furthermore, we showed that there is a simple scaling of the adsorption energies with the radius of the SWNT. We believe that the tunable adsorption can have important implications for metal coverage and selective adsorption of foreign atoms and molecules on the carbon nanotubes, and can lead to a wide variety of technological applications, ranging from hydrogen storage to new materials [8].
PACS numbers: 73.22.-f, 68.43.Bc, 68.43.Fg

We have investigated the electronic energy structure and charge density of bare and single atom adsorbed SWNTs with and without radial strain by performing first-principle density functional calculations [9]. We expressed the wave functions by linear combinations of plane waves up to an energy cutoff of $500 \mathrm{eV}$ within the tetragonal supercell geometry (separation between the tubes is taken to be $7.5 \AA$ and $c$ is equal to the lattice constant of SWNT along its axis). With this energy cutoff and using ultrasoft pseudopotentials for carbon [10] the total energies of nanotube-adatom systems converge within $0.5 \mathrm{meV} /$ atom. The Monkhorst-Pack [11] special k-point scheme is used with $0.02 \AA^{-1} k$-point spacing resulting in $5 \mathbf{~ k}$-points along the tube axis. Results have been obtained within the generalized gradient approximation [12] for fully relaxed geometries including all carbon and adsorbate positions and the lattice constant of the tube along the $z$ axis.

To investigate the effect of the radial deformation we first consider adsorption of $\mathrm{H}$ and a simple metal, $\mathrm{Al}$, on undeformed SWNTs. In Fig. 1, we present the binding energies of $\mathrm{H}$ and $\mathrm{Al}$ adsorbed on the $(n, 0)$ zigzag nanotubes calculated for $n=7,8,9,10,12$. $\mathrm{H}$ is adsorbed at the top site, i.e., directly above the $\mathrm{C}$ atoms of the tube, $\mathrm{Al}$ favors

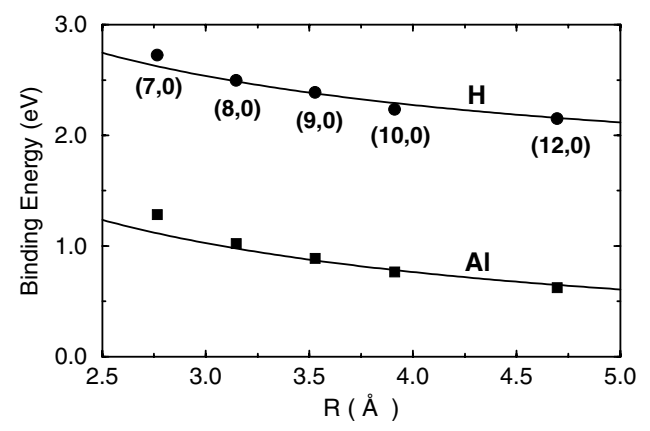

FIG. 1. Binding energies $E_{b}$ of single hydrogen and aluminum atom adsorbed on the zigzag SWNTs versus the radius of the tubes. The solid line is the fit to $E_{b}=E_{o}+C / R$ (see text). 
the hollow site, i.e., above the center of the hexagon as in the graphite surface. The binding energy $E_{b}$,

$$
E_{b}=E_{T}[\mathrm{SWNT}]+E_{A}-E_{T}[\mathrm{SWNT}+A],
$$

is calculated in terms of the total energy of SWNT, $E_{T}[\mathrm{SWNT}]$, total energy of SWNT with an adsorbed atom $A, E_{T}[\mathrm{SWNT}+A]$, and the energy of the single, free atom, $E_{A}$. Here the bare SWNT and SWNT with an adatom $A$ are free of external stress, and all the atomic coordinates are fully relaxed. Moreover, since $E_{b}$ is calculated by using the same supercell, the spurious adatom-adatom interaction along the tube axis is substracted. The positive value of $E_{b}$ indicates that the adsorption is exothermic and hence stable. It is found that the binding energy of an adatom decreases with increasing radius (or decreasing curvature) of the tube, and eventually saturates at a value corresponding to that on graphene plane. Hence the variation of binding energies of $\mathrm{H}$ and Al with the radius of the zigzag tube fits to the curve given by the expression,

$$
E_{b, A}(R)=E_{o, A}+\frac{C_{A}}{R},
$$

where $E_{o, A}$ is the binding energy of the adatom $A(\mathrm{H}$ or $\mathrm{Al})$ on the graphene plane. We calculated $E_{o, \mathrm{H}}=1.49 \mathrm{eV}$ and $E_{o, \mathrm{Al}}=-0.02 \mathrm{eV}$. Interestingly, the fitting parameter $C_{A}$ is found to be independent of the adatom $(\mathrm{H}$ and $\mathrm{Al})$ and is equal to $\sim 3.14 \mathrm{eV} \AA$. Currently, we are extending our calculations to see if this relation holds for other adsorbates as well. The binding energies calculated for $(n, 0)$ SWNTs with $n<8$ deviate from the above simple scaling perhaps due to the fact that the singlet $\pi^{*}$ band, which is normally in the conduction band, falls into the band gap as a result of increased $\sigma^{*}-\pi^{*}$ mixing at high curvature [7,13]. Note that, while the band gap shows significant change with $n$ [for example, upon going from $(8,0)$ to $(9,0) E_{g}$ changes from $0.65 \mathrm{eV}$ to $0.09 \mathrm{eV}$ ], the binding energies vary smoothly with $R^{-1}$. Increasing $E_{b}$ with decreasing $R$ (or with decreasing $n$ ) shows that for small $R$ the character of the surface deviates from that of the graphene. This finding also suggests that by creating regions of different curvature on a single SWNT by radial deformation one can attain different values of binding energies.

The radial deformation that we consider in this study is generated by applying uniaxial compressive stress on a narrow strip on the surface of the SWNT [7]. In practice such a deformation can be realized by pressing the tube between two rigid flat surfaces. The radius is decreased in the $y$ direction, while it is elongated along the $x$ direction. As a result, the circular cross section is distorted to an elliptical one with major and minor axes, $a$ and $b$, respectively. The elliptical radial deformation can be described by the magnitude of the applied strain, and it is defined as $\epsilon_{y y}=(R-b) / R$ where $R$ is the radius of the undeformed nanotube (with zero strain). For different values of $\epsilon_{y y}$ we carried out full structural optimization under the constraint that the minor axis is kept fixed at a preset value by freezing the carbon atoms at both ends of the minor axis [7]. Then, under this constraint, the coordinates of the adatom and remaining carbon atoms and the lattice constant of the tube, $c$, are optimized until there remain only forces opposite to the applied strain on the fixed atoms, but all other force components are less than $0.01 \mathrm{eV} / \AA$.

Figure 2a shows the variation of the binding energy $E_{b}$ of a single hydrogen atom adsorbed on the $(8,0)$ surface with the applied radial strain for two cases: (i) The binding energy of a single $\mathrm{H}$ atom adsorbed on the high curvature side of the surface (i.e., specified as the sharp site near one of the ends of the major axis, $x=a, y=0$ ) traces the upper curve in Fig. 2a. (ii) The lower curve is for the adsorption on the low curvature side near one of the ends of the minor axis (i.e., $x=0, y=b$ specified as the flat site). Here the binding energy is defined as in Eq. (1), except that $E_{T}[\mathrm{SWNT}]$ and $E_{T}[\mathrm{SWNT}+A]$ are calculated for radially deformed SWNTs. The binding energy of $\mathrm{H}$ adsorbed on the high curvature (sharp) site is increased by $0.85 \mathrm{eV}$ for $\epsilon_{\mathrm{yy}}=0.3$. On the other hand, $E_{b}$ for the adsorption on the low curvature (flat) site first decreases with increasing $\epsilon_{y y}$, and then saturates at an energy $0.25 \mathrm{eV}$ less than that corresponding to $\epsilon_{\mathrm{yy}}=0.0$. The difference of binding energies of the sharp and flat sites, $\Delta E_{b}$, is substantial and is equal to $\sim 1.1 \mathrm{eV}$. This
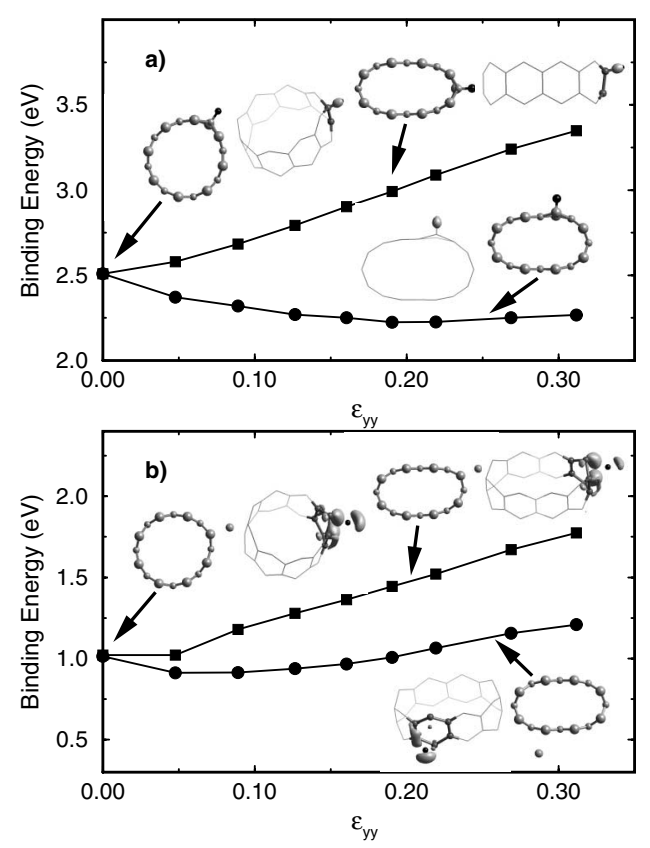

FIG. 2. (a) Variation of the binding energy $E_{b}$ of a single hydrogen atom adsorbed on a $(8,0)$ zigzag SWNT as a function of the elliptic radial deformation, $\epsilon_{y y}$. The upper curve corresponds to $\mathrm{H}$ adsorbed on the high curvature region near the end of the major axis $a$ (sharp site). The lower curve is for the adsorption on the low curvature region at the end of the minor axis ( flat site). (b) Same as (a) for a single adsorbed Al atom. Insets: Ball and stick models and isosurface plot of difference charge densities, $\Delta \rho$ for each case. (See text.) 
value is $44 \%$ of the binding energy of $\mathrm{H}$ on the undeformed SWNT. As a result of $\mathrm{H}$ adsorption, the $s p^{2}$ character of the bonding of the tube has changed locally and become more like $s p^{3}$. The lengths of the C-C bonds at the close proximity of $\mathrm{H}$ have increased slightly.

The binding energy of $\mathrm{Al}$ shown in Fig. $2 \mathrm{~b}$ exhibits a behavior similar to that of $\mathrm{H}$, despite $\mathrm{H}$ and $\mathrm{Al}$ favoring different sites on the $(8,0)$ tube: $E_{b}$ at the sharp site of the deformed SWNT increases with increasing $\epsilon_{y y}$. For example, $E_{b}$ increases by $\sim 0.80 \mathrm{eV}$ for $\epsilon_{y y}=0.3$ which is $80 \%$ of the binding energy on the undeformed tube. For Al absorbed on the flat site, $E_{b}$ first decreases with increasing $\epsilon_{y y}$, then gradually increases. Adsorption of $\mathrm{Al}$ induces local changes in the atomic and electronic structures. For example, the surface of the tube where $\mathrm{Al}$ is adsorbed expands.

The variation of $E_{b}$ with the radial deformation is consistent with the results illustrated in Fig. 1. In general, the higher is the curvature under deformation, the higher the binding energy. The effect of the elastic deformation is further investigated by analyzing the charge density and electronic structure. The Mulliken analysis estimates that $\sim 0.37$ electrons is transferred from $\mathrm{H}$ to carbon [14]. The $\mathrm{C}-\mathrm{H}$ bond is directional and is covalent with a partial ionic component. The charge difference, $\Delta \rho=\rho[\mathrm{SWNT}+H]-\rho[\mathrm{SWNT}]-\rho[\mathrm{H}]$, which is calculated in terms of the undeformed (or deformed) SWNT with $\mathrm{H}$ (adsorbed at different sites), undeformed (deformed) clean SWNT, and single $\mathrm{H}$, is shown in the inset of Fig. 2a. $\Delta \rho$ indicates no significant change with radial deformation. On the other hand, the charge transfer upon the adsorption of $\mathrm{Al}$ is different from $\mathrm{H}$. Since $\mathrm{Al}$ is adsorbed on the center of a hexagon, the bond between $\mathrm{Al}$ and SWNT is distributed to nearest $\mathrm{C}$ atoms with charge accumulation between $\mathrm{Al}$ and those $\mathrm{C}$ atoms. The bond charge slightly increases at the sharp site as shown in the inset of Fig. 2b. The charge transfer from the $\mathrm{Al}$ atom is estimated to be 0.71 electrons.

Explanation of this remarkable and significant change of the binding energy with radial deformation is sought in the electronic energy structure and the total and partial density of states. The adsorption of $\mathrm{H}$ gives rise to a new state which falls in the gap at $\Gamma$ and coincides with $E_{F}$. This state partially overlaps with the conduction band when $\mathrm{H}$ is adsorbed at the flat site, whereas it occurs near the valence band edge at the sharp site. A similar situation occurs with the adsorption of $\mathrm{Al}$ as illustrated in Fig. 3. The band gap is wide open, and a resonant state $\sim 2.5 \mathrm{eV}$ below $E_{F}$ and a $p$-derived adsorption state which sets $E_{F}$ falls at the center of the gap for the adsorption of $\mathrm{Al}$ on the undistorted SWNT [15]. In the case of adsorption on the flat site the latter state overlaps the conduction band (Fig. 3c), but it dips into the valence band at the sharp site (Fig. 3d). Accordingly, the sharp-site adsorption involves the valence band states of SWNT, while the conduction band states are involved in the flat site adsorption.

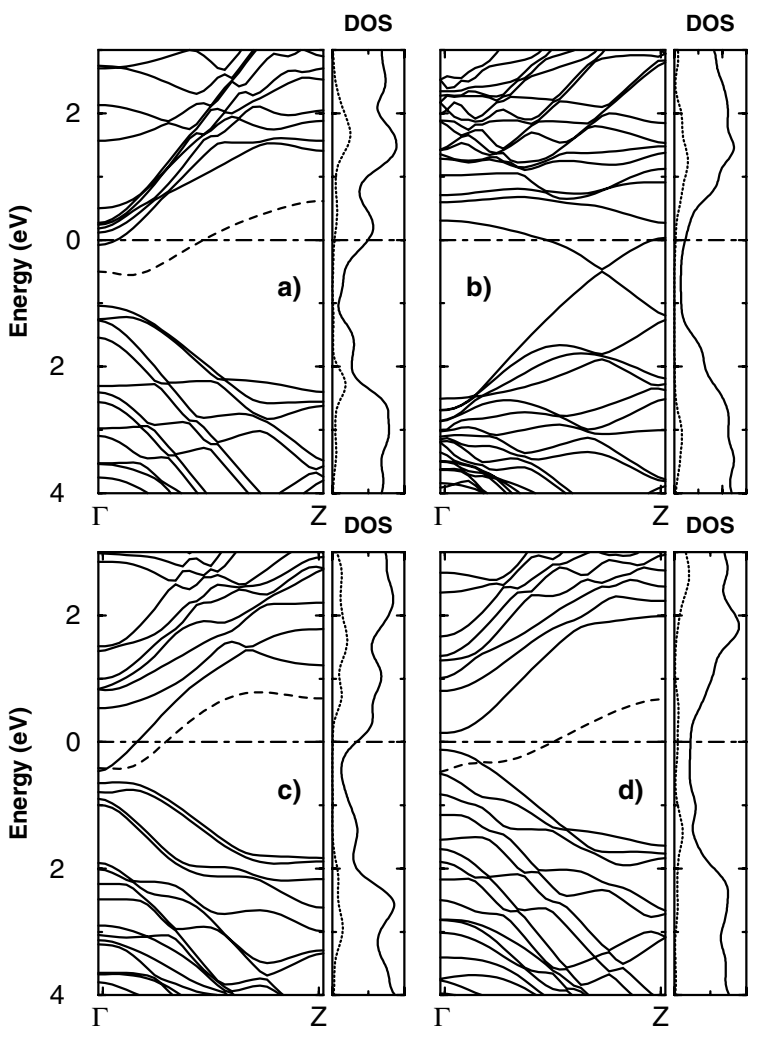

FIG. 3. Electronic band structure, total and partial density of states (with dotted lines) of Al atom adsorbed on a SWNT. The zero of energy is taken at the Fermi level shown by dash-dotted lines. The band due to $\mathrm{Al}$ in the band gap is shown by the dashed line. Al adsorbed: (a) on the undeformed $(8,0)$ tube, (b) on the undeformed $(6,6)$ tube, (c) on the flat site of the $(8,0)$ tube, $(d)$ on the sharp site of the $(8,0)$ tube. $\epsilon_{y y}=0.31$ for (c) and (d).

The above argument is in accord with the binding energies calculated for the adsorption of $\mathrm{Al}$ on the undeformed and deformed $(6,6)$ armchair SWNT. Note that the $(6,6)$ as well as all $(n, n)$ SWNTs are metallic with finite $\mathcal{D}\left(E_{F}\right)$ owing to the $\pi$ and $\pi^{*}$ states crossing and setting $E_{F}$. Our calculations show that $\mathcal{D}\left(E_{F}\right)$ of the $(6,6)$ decreases slightly with increasing radial deformation, but it remains essentially finite. We found $E_{b}=0.91 \mathrm{eV}$ for the adsorption of $\mathrm{Al}$ on the $(6,6)$ SWNT. Upon the deformation of the tube with $\epsilon_{\mathrm{yy}}=0.22, E_{b}$ corresponding to sharpand flat-site adsorption has changed to 0.95 and $0.85 \mathrm{eV}$, respectively. Therefore, the difference between the binding energies of the sharp- and flat-site adsorption is only $0.1 \mathrm{eV}$, and hence is negligible as compared to the situation above discussed for the $(8,0)$ SWNT. In Fig. $3 b$ the state associated with the adsorption of $\mathrm{Al}$ on the undeformed $(6,6)$ tube occurs above the Fermi level and donates its electron to the metallic $\pi$ and $\pi^{*}$ bands. It appears that $E_{b}$ is almost pinned by the above mechanism.

Radial deformation induced changes in the energies of valence band edge (VBE) and conduction band edge (CBE), as well as the adsorption state (AE) at the $\Gamma$-point 


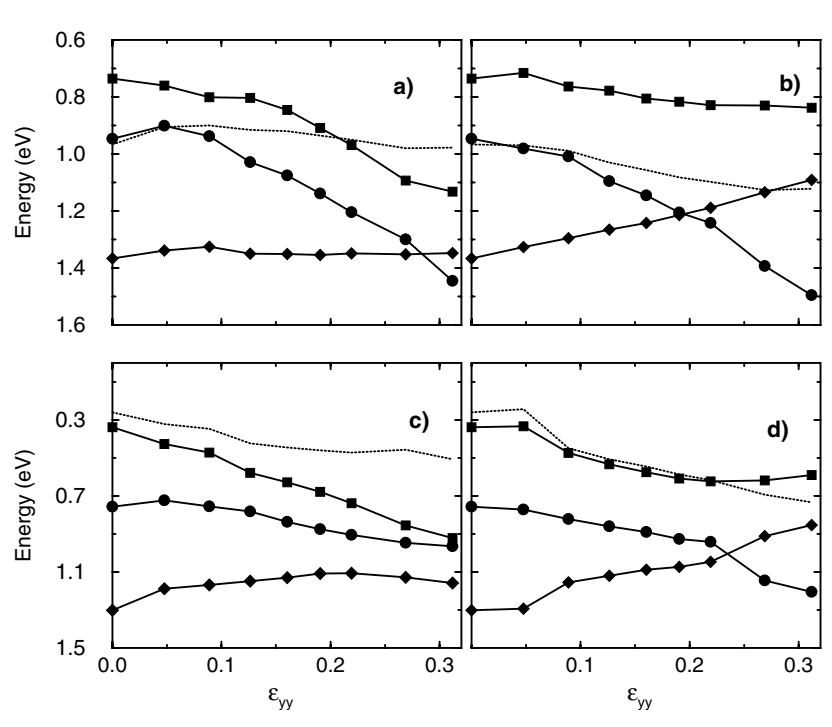

FIG. 4. The shifts of band edges at $\Gamma$-point with radial deformation on a $(8,0)$ tube. $\mathrm{H}$ adsorbed: (a) on the flat site, (b) on the sharp site; Al adsorbed: (c) on the flat site, (d) on the sharp site. The valence band edge, the conduction band edge, the adsorption state, and the Fermi energy are shown by diamonds, squares, circles, and dots, respectively.

illustrated in Fig. 4 corroborate the above arguments. In the case of adsorption on the flat site, VBE is almost constant for both $\mathrm{H}$ and $\mathrm{Al}$, while $\mathrm{CBE}$ and $\mathrm{AE}$ both shift downwards. As a result of this, more conduction band states contribute to the bond energy. By contrast, for adsorption on the sharp site, while AE behaves very similarly to the previous case, $\mathrm{CBE}$ is now approximately constant, but VBE shifts upwards. In this situation, the contribution to the bond energy from conduction band states is decreased.

In conclusion, we showed that the chemical reactivity of a zigzag SWNT can be modified reversibly and variably by the radial deformation. The effect of deformation is significantly different for the zigzag and armchair SWNTs. It is remarkable that $\mathrm{Al}$, which is not bound to the graphite surface, can be adsorbed at a high curvature site of a zigzag SWNT under radial deformation with a binding energy of $1.8 \mathrm{eV}$. This novel property may have important implications for various chemical and electronic applications, such as selective absorption and desorption of molecules and atoms, fragmentation and chemical sensors, magnetic tubes, etc.

This work was partially supported by the NSF under Grant No. INT97-31014 and TÜBÍTAK under Grant No. TBAG-1668(197 T 116). S.C. thanks Professor S. Süzer for stimulating discussions.

[1] S. Iijima, Nature (London) 354, 56 (1991).

[2] M.S. Dresselhaus, G. Dresselhaus, and P.C. Eklund, Science of Fullerenes and Carbon Nanotubes (Academic Press, San Diego, 1996); R. Saito, G. Dresselhaus, and M.S. Dresselhaus, Physical Properties of Carbon Nanotubes (Imperial College Press, London, 1998); N. Hamada, S. Sawada, and A. Oshiyama, Phys. Rev. Lett. 68, 1579 (1992).

[3] A. Bezryadin, A. R. M. Verschueren, S. J. Tans, and C. Dekker, Phys. Rev. Lett. 80, 4036 (1998).

[4] A. Rochefort, P. Avouris, F. Lesage, and D. R. Salahub, Chem. Phys. Lett. 297, 45 (1998).

[5] C. J. Park, Y. H. Kim, and K. J. Chang, Phys. Rev. B 60, 10656 (1999).

[6] Ç. Kılıç, S. Ciraci, O. Gülseren, and T. Yildirim, Phys. Rev. B 62, R16345 (2000).

[7] O. Gülseren, T. Yildirim, S. Ciraci, and Ç. Kılıç (to be published).

[8] A. C. Dillon et al., Nature (London) 386, 377 (1997); C. Liu et al., Science 286, 1127 (1999); Q. Wang et al., Phys. Rev. Lett. 82, 956 (1999); M. C. Gordillo, J. Boronat, and J. Casulleras, Phys. Rev. Lett. 85, 2348 (2000); M. M. Calbi, F. Toigo, and M. W. Cole, Phys. Rev. Lett. 86, 5062 (2001).

[9] M. C. Payne et al., Rev. Mod. Phys. 64, 1045 (1992).

[10] D. Vanderbilt, Phys. Rev. B 41, 7892 (1990).

[11] H. J. Monkhorst and J. D. Pack, Phys. Rev. B 13, 5188 (1976).

[12] J. P. Perdew and Y. Wang, Phys. Rev. B 46, 6671 (1992).

[13] X. Blase, L. X. Benedict, E. L. Shirley, and S. G. Louie, Phys. Rev. Lett. 72, 1878 (1994).

[14] T. Yildirim, O. Gülseren, and S. Ciraci, Phys. Rev. B 64, 075404 (2001).

[15] Note that the band associated with the adsorption state in the band gap displays a small dispersion for $\mathrm{Al}$ but practically no dispersion for $\mathrm{H}$. This is the artifact of the supercell geometry used in the present study. 